\title{
Generation of picosecond and subpicosecond light pulses with saturable absorbers
}

\author{
A. PENZKOFER \\ Physik-Department der Technischen Hochschule München, Munich, Germany
}

\section{Received 3 September 1973}

\begin{abstract}
Single light pulses, generated by a mode-locked Nd-glass laser, were shortened with saturable absorbers of low initial transmission $T_{0}$. The pulse duration was reduced from 8 to $2.6 \mathrm{ps}$ after a single pass through a dye cell of $T_{0}=10^{-7}$. Light pulses as short as 0.5 ps were observed after five transits through an absorberamplifier system. Detailed calculations of the stationary and the transient situation (with respect to the dye relaxation time $\tau$ ) are presented to demonstrate optimum conditions for the pulse shortening.
\end{abstract}

\section{Introduction}

In conventional mode-locked Nd-glass lasers, light pulses with durations of 5 to 10 ps are generated $[1,2]$. This duration is considerably longer than expected from the theoretical limit of $\Delta t_{\text {min }} \simeq 1 / \Delta \nu_{\mathrm{L}} \simeq 10^{-13} \mathrm{~s}\left(\Delta \tilde{\nu}_{\mathrm{L}} \simeq 200 \mathrm{~cm}^{-1}\right.$ [3] is the fluorescence line width of the Nd-glass). There are several factors responsible for the observed long pulse durations in passively mode-locked Nd-glass lasers:

(i) the spectral narrowing in the linear amplification phase of the pulse development increases the pulse duration [4];

(ii) the finite de-excitation time $\tau$ of the saturable absorber $(\tau \simeq 9 \mathrm{ps})$ reduces the pulse shortening effect in the non-linear range of the dye $[5,6]$;

(iii) the effects of self-phase modulation [7, 8] and dispersion [9] act together to reduce the pulse shortening of the non-linear absorber at the beginning of the pulse train; they broaden the pulses in the middle of the pulse train and they cause pulses with subpicosecond substructure at the end of the pulse train [10].

With non-linear methods, picosecond pulses can be shortened, or new pulses of shorter duration can be produced. Optical compression of frequency-modulated pulses allows the generation of very short light pulses [11, 12]; a shortening from 5 to 0.4 ps has been reported [11]. In a Raman oscillator experiment-driven with a picosecond pulse train - Raman pulses with durations as short as one tenth of the generating pulses have been obtained [13].

In this paper we describe a method of shortening single picosecond light pulses using saturable dyes of low transmission [14]. In Section 2 the pulse shortening mechanism is discussed and in Section 3 a series of calculated data are compared with experi- 
mental results. Pulse shortening is studied with single passes through an absorber cell and by multiple transits through an absorber-amplifier system. In the last section the limitations of this method of pulse shortening are discussed.

\section{Passage of a light pulse through a saturable absorber}

When a light pulse traverses a cell containing a saturable dye a certain amount of light is absorbed by the dye molecules (electric dipole transition) and the molecules are excited from the ground state $\mid a>$ to the level $\mid b>$ (see Fig. 1). The energy difference between $\mid a>$ and $\mid b>$ is $h \nu_{\mathrm{L}}$ ( $\nu_{\mathrm{L}}$ is the laser frequency). At low intensities $I$, the

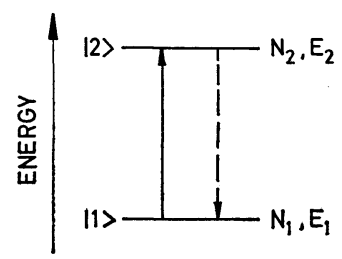

Figure 1 Two-level system for a saturable dye. $N_{1}$ and $N_{2}$ are the numbers of molecules [ $\mathrm{cm}^{-3}$ ] in ground state $\mid 1>$ and excited state $\mid 2>$, respectively. The energy difference $E_{2}-E_{1}$ is equal to the laser photon energy $h \nu_{\mathrm{L}}$.

absorption is intensity-independent. With increasing input intensity the population $N_{2}$ of the excited level $\mid b>$ increases and approaches the same value as the population $N_{1}$ of the ground state $\mid a>$ at high light intensities. Under these conditions the transmission of light through the non-linear absorber increases with intensity and approaches unity at high laser intensity: the absorption decreases due to the reduced population $N_{1}$ of the ground state (absorption proportional $N_{1}$ ), and the induced emission increases with the population $N_{2}$ of the excited state (induced emission proportional $N_{2}$; absorption coefficient $\alpha$ is proportional to $N_{1}-N_{2}$ ). The excited molecules relax to the ground state by spontaneous emission and by radiationless transitions (relaxation time $\tau$ ). In the regime of intensity-dependent absorption, the leading and trailing parts of the light pulse are more reduced than the centre of the pulse; this fact leads to a shortening of the pulse.

The interaction of light with a saturable dye can be described by a two-level system [15] (see Fig. 1). When the duration of the light pulses $\Delta t$ is longer than the dephasing time $T_{2}$ of the dye the following rate equations hold:

$$
\begin{gathered}
\frac{\mathrm{d} N_{1}}{\mathrm{~d} t}=-\frac{I \sigma}{h \nu_{\mathrm{L}}}\left(N_{1}-N_{2}\right)+\frac{N_{2}}{\tau} \\
\frac{\mathrm{d} N_{2}}{\mathrm{~d} t}=\frac{I \sigma}{h \nu_{\mathrm{L}}}\left(N_{1}-N_{2}\right)-\frac{N_{2}}{\tau} \\
\frac{\mathrm{d} I}{\mathrm{~d} t}=\frac{\partial I}{\partial t}+\frac{c}{\eta} \frac{\partial I}{\partial x}=-\frac{c}{\eta} I \sigma\left(N_{1}-N_{2}\right),
\end{gathered}
$$

where $\sigma$ is the absorption cross-section, $c$ the velocity of light and $\eta$ the refractive index. 
The first part on the right hand side of Equations 1 and 2 describes the absorption and induced emission while the second part corresponds to the spontaneous emission and the non-radiative relaxation. Equation 3 relates the change of pulse intensity to absorption and to induced emission. Introducing $n=N_{1}-N_{2}$ and $N=N_{1}+N_{2}$, Equations 1 to 3 can be rewritten:

$$
\begin{gathered}
\frac{\mathrm{d} n}{\mathrm{~d} t}=-\frac{2 I}{h \nu_{\mathrm{L}}} n+\frac{N-n}{\tau} \\
\frac{\partial I}{\partial t}+\frac{c}{\eta} \frac{\partial I}{\partial x}=-\frac{c}{\eta} I \sigma n .
\end{gathered}
$$

In the stationary case where the duration of the incident pulse $\Delta t_{\mathrm{in}} \gg \tau$ [16], the time derivatives in Equations 4 and 5 are zero and one obtains the implicit solution [17]:

$$
T=T_{\mathrm{o}} \exp \left\{\frac{I_{\text {in }}}{I_{\mathrm{s}}}(1-T)\right\} .
$$

$T=I_{\mathrm{tr}} / I_{\mathrm{in}}=I(z=\ell) / I(z=0)$ is the intensity transmission of the non-linear absorber; $T_{0}=\exp \left(-\alpha_{0} \ell\right)=\exp (-\sigma N \ell)$ is the transmission through the dye cell at low input intensity $I_{\text {in }}\left(\ell\right.$, cell length); and $I_{\mathrm{S}}=h \nu_{\mathrm{L}} /(2 \sigma \tau)$ is called the saturation intensity. $I_{\mathrm{s}}$ is defined as the intensity value where the absorption coefficient $\alpha=\sigma n$ is reduced to half its linear value (i.e. $\alpha=\alpha_{0} / 2, n=N / 2$ ). Equation 6 clearly shows that $T$ is always larger than $T_{\mathrm{o}}$ and that it approaches unity at high values of $I_{\mathrm{in}}$. As a result, the light pulses are shortened because the transmission is small for the wings and large for the central part of the pulses [18].

When the duration of the incident pulse $\Delta t_{\text {in }}$ (FWHM) is comparable to or shorter than the relaxation time $\tau$, Equations 4 and 5 can be rewritten as an integral equation [19]:

$$
\begin{aligned}
& T\left(T_{\mathrm{o}}, I_{\mathrm{o}} \times s\left(t^{\prime}, r^{\prime}\right), \frac{\tau}{\Delta t_{\mathrm{in}}}\right)=T_{\mathrm{o}} \exp \left\{\frac{2 \sigma I_{\mathrm{o}}}{h \nu_{\mathrm{L}}} \int_{-\infty}^{t^{\prime}} s\left(t^{\prime \prime}, r^{\prime}\right)\right. \\
\times & {\left.\left[1-T\left(T_{0}, I_{\mathrm{o}} \times s\left(t^{\prime \prime}, r^{\prime}\right), \frac{\tau}{\Delta t_{\mathrm{in}}}\right)\right] \exp \left(\frac{t^{\prime \prime}-t^{\prime}}{\tau}\right) \mathrm{d} t^{\prime \prime}\right\}, }
\end{aligned}
$$

This equation shows that the intensity transmission $T$ depends on the initial (low intensity) transmission $T_{0}$, on the input intensity $I_{\mathrm{in}}=I_{0} \times s\left(t^{\prime}, r^{\prime}\right)$ and on the ratio $\tau / \Delta t_{\text {in }} . I_{0}$ is the peak intensity and the function $s\left(t^{\prime}, r^{\prime}\right)=s\left((t-\eta z / c) / \Delta t_{\text {in }}, r / \Delta r_{\text {in }}\right)$ gives the temporal and spatial shape of the incident pulse. Equation 7 indicates that $T$ starts with $T_{0}\left(\right.$ at $\left.t^{\prime}=-\infty\right)$, increases with time as long as the integral grows and decreases at the end of the pulse due to the exponential decay factor in the integral. At high peak intensity the transmission $T$ approaches unity (preferentially in the central part of the pulse). Two mechanisms are responsible for the pulse shortening in the transient case: (i) The leading part of the pulse is strongly absorbed since - at the beginning - all the dye molecules are in their ground states. (ii) When a certain amount 
of energy is absorbed from the leading part of the pulse the population $N_{2}$ of the excited level $|b\rangle$ approaches its intensity-dependent equilibrium and the transmission becomes similar to the stationary case. For intense pulses which bleach the dye the energy per cross-section absorbed from the leading part is $E / A \simeq h \nu_{\mathrm{L}} N / / 2=$ $-h \nu_{\mathrm{L}} \ln \left(T_{\mathrm{o}}\right) /(2 \sigma) ;\left(\mathrm{e} . \mathrm{g} .: E / A \simeq 8 \mathrm{~mJ} \mathrm{~cm}{ }^{-2}\right.$ for $T_{\mathrm{o}}=10^{-7}$ and $\left.\sigma=1.84 \times 10^{-16} \mathrm{~cm}^{2}\right)$.

In the following section numerical solutions of Equation 7 will be presented for a variety of parameters which are relevant for experimental situations. To elucidate our calculations immediate comparison with experimental observations will be made.

\section{Comparison of theory and experiments}

\subsection{Single pass system}

We studied the shortening of single picosecond light pulses when the pulse passed through a dye cell of well defined initial transmission. In Fig. 2 our experimental set-up is depicted schematically. A single, bandwidth limited, pulse of $\Delta t_{\mathrm{in}} \simeq 8 \mathrm{ps}$ was selected from a pulse train of a mode-locked Nd-glass laser. The single pulse was amplified

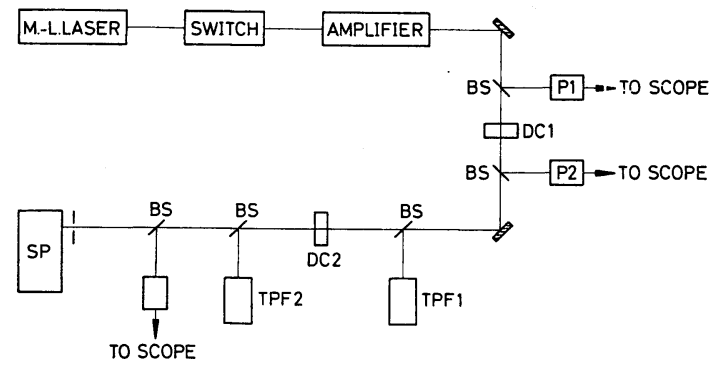

Figure 2 Experimental set-up for single pass system. Saturable absorber cell DC2 $\left(T_{0}=10^{-3}\right.$ or $\left.10^{-7}\right)$; Beam splitters BS; photodetectors P1, P2, P3; two-photon fluorescence systems TPF1, TPF2; spectrograph SP. The input peak intensity $I_{0}$ is monitored by the photodetectors P1, P2 and the dye cell DC1 $\left(T_{0}=0.173\right)$.

before traversing the dye cell DC2 with transmission $T_{0}$. The peak intensity of the incident pulse was determined by a technique described in reference [20]; the ratio of the two signals of the photocells $\mathrm{P}_{1}$ and $\mathrm{P}_{2}$ allow to determine the absolute value of $I_{0}$ with an accuracy of better than $\pm 20 \%$. The pulse duration of the incident pulse $\Delta t_{\text {in }}$ (before DC2) and of the transmitted pulse $\Delta t_{\text {out }}$ (behind DC2) were measured with two photon fluorescence systems TP1 and TP2 [21], respectively. In addition, the spectrometer SP allows a determination of the spectrum of the transmitted pulse.

Calculated transmitted pulse shapes are depicted in Fig. 3. The normalized intensity $I_{\mathrm{tr}}(t-\eta z / c) / I_{0}$ is plotted versus normalized time for several input peak intensities $I_{0}$ of a Gaussian input pulse (broken line) of 8 ps duration. The initial transmission of the dye is chosen to be $T_{0}=10^{-7}$ (for dye parameters see [16]). Pulses with high peak intensity $\left(I_{0} \gtrsim 5 \times 10^{9} \mathrm{~W} \mathrm{~cm}^{-2}\right)$ are strongly absorbed at the leading edge until the dye is excited to its stationary equilibrium. The centre of the pulse bleaches the dye and the trailing part of the pulse is reduced by the stationary intensity-dependent transmission of the dye. In this case the pulse shortening effect is small. At very low peak 


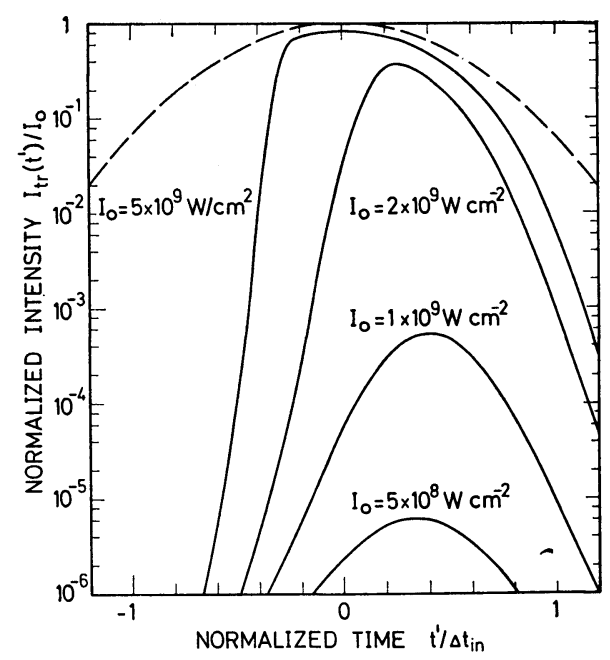

Figure 3 Change of pulse intensity versus time after a single pass through a saturable absorber. Broken line: normalized intensity of the input pulse ( $\Delta t_{\text {in }}=8 \mathrm{ps}$, Gaussian pulse shape). Solid curves: normalized intensities of the transmitted pulse for four values of the input peak intensity $I_{0}$. Dye parameters: $T_{0}=10^{-7}, \sigma=1.84 \times 10^{-16} \mathrm{~cm}^{2}, \tau=9.1 \mathrm{ps}$.

intensity $\left(I_{0}<10^{9} \mathrm{~W} \mathrm{~cm}^{-2}\right)$ only a small portion of the dye molecules is excited, the whole pulse is strongly absorbed and again pulse shortening is not significant. Of interest is the intensity range around $I_{0}=2 \times 10^{9} \mathrm{~W} \mathrm{~cm}^{-2}$ where the pulse shortening effect is optimum; in this case the peak intensity is lowered by a factor of 2 to 10 only.

The calculated pulse shortening ratio $\Delta t_{\mathrm{tr}} / \Delta t_{\text {in }}$ versus input peak intensity $I_{\mathrm{o}}$ is depicted in Fig. 4 for several initial transmissions $T_{0}$. The duration of the incident Gaussian pulse is $\Delta t_{\mathrm{in}}=8 \mathrm{ps}$ in Fig. $4 \mathrm{a}$ and $\Delta t_{\mathrm{in}}=4 \mathrm{ps}$ in Fig. $4 \mathrm{~b}$. The figures show as discussed above - that the pulse shortening effect is small at low and very high intensities. An optimum intensity value $I_{\mathrm{o}, \text { opt }}$ exists for each value of $T_{\mathrm{o}}$ at which pulse shortening is most effective. $I_{0}$,opt shifts to higher intensity values as $T_{0}$ is lowered.

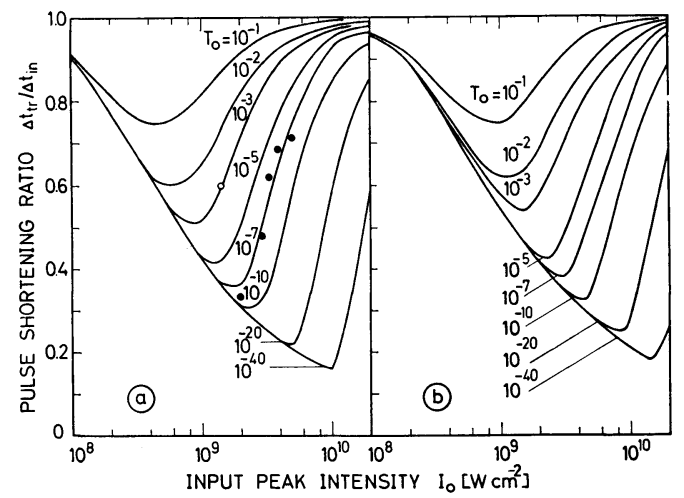

Figure 4 Pulse shortening ratio $\Delta t_{\mathrm{tr}} / \Delta t_{\text {in }}$ versus input peak intensity $I_{0}$ for several values of initial dye transmission $T_{0}$ (single pass). Input pulse: Gaussian shape, duration $\Delta t_{\text {in }}=8 \mathrm{ps}$ (a) and $\Delta t_{\text {in }}=4 \mathrm{ps}(\mathrm{b})$. Note the experimental points for $T_{0}=10^{-3}$ (open circle) and $T_{0}=10^{-7}$ (closed circles). 
Experiments were carried out with $\Delta t_{\text {in }} \simeq 8 \mathrm{ps}$ and initial transmission of the dye of $T_{\mathrm{o}}=10^{-3}$ and $10^{-7}$. The measured points are added to Fig. 4a. The intensity dependence of the pulse shortening ratio was determined for $T_{0}=10^{-7}$. All experimental points are in good agreement with calculated curves.

A comparison of Figs. $4 \mathrm{a}$ and $\mathrm{b}$ shows that pulses of $\Delta t_{\mathrm{in}}=4 \mathrm{ps}$ are nearly as effectively shortened as pulses of $\Delta t_{\text {in }}=8 \mathrm{ps}$. At fixed $T_{\mathrm{o}}$ the optimum intensity values $I_{0}$,opt shift slightly to higher numbers for smaller $\Delta t_{\text {in. }}$. Fig. 5 shows the shortening ratio versus input peak intensity for the three significant cases: $\Delta t_{\text {in }} / \tau \gg 1$ (steady state), $\Delta t / \tau=1$ and $\Delta t / \tau=0.1$. Gaussian input pulses and $T_{0}=10^{-7}$ are assumed in these cal-

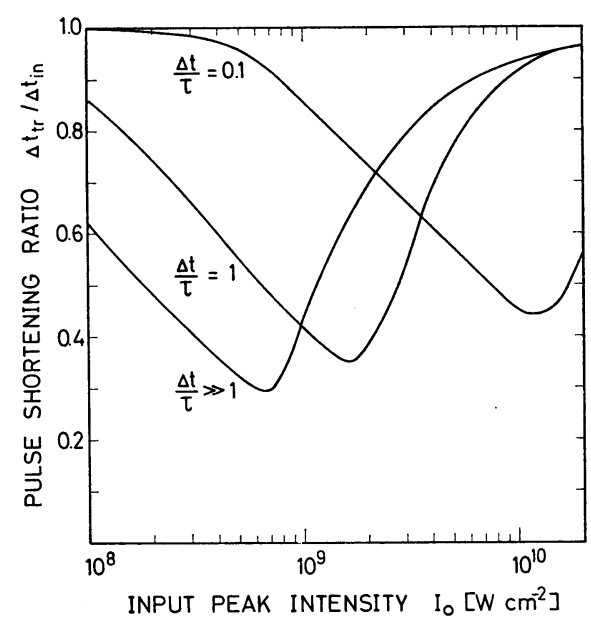

Figure 5 Pulse shortening ratio $\Delta t_{\mathrm{tr}} / \Delta t_{\mathrm{in}}$ versus input peak intensity $I_{0}$ for several ratios of input pulse duration to dye relaxation time, $\Delta t_{\mathrm{in}} / \tau$. (Gaussian shape of the incident pulse, $T_{0}=10^{-7}$, $\sigma=1.84 \times 10^{-16} \mathrm{~cm}^{2}$.)

culations. The pulses are most effectively shortened in the steady state case, but the pulse shortening effect is still pronounced when the pulse duration is one tenth of the relaxation time of the saturable absorber. The intensity range for optimum pulse shortening, shifts to higher values when the pulse duration becomes shorter.

The calculations discussed so far were concerned with pulses of Gaussian shape. We now wish to consider the pulse shortening ratio for other pulse shapes. In the stationary case a rectangular pulse is not shortened since the pulse experiences the same absorption for its full length. Pulses with Gaussian, hyperbolic secant and Lorentzian pulse shape show distinct pulse shortening in the stationary case with shortening ratios nearly independent of the individual pulse shape (see Fig. 6 for $T_{0}=10^{-7}$ ). A transient situation is depicted in Fig. $7\left(\Delta t_{\mathrm{in}} / \tau=0.88\right.$ and $\left.T_{\mathrm{o}}=10^{-7}\right)$. In this example the rectangular pulse is most effectively shortened while Gaussian, hyperbolic secant and Lorentzian pulses show quite a similar pulse shortening ratio.

Pulse shortening by a saturable absorber reduces the peak intensity of a pulse. Figs. $8 \mathrm{a}$ and $\mathrm{b}$ show the reduction of normalized peak intensity $I_{\mathrm{tr}, \max } / I_{\mathrm{o}}$ versus input peak intensity $I_{\mathrm{o}}$ for various $T_{\mathrm{o}}$ values at $\Delta t_{\mathrm{in}}=8 \mathrm{ps}$ and $\Delta t_{\mathrm{in}}=4 \mathrm{ps}$, respectively 


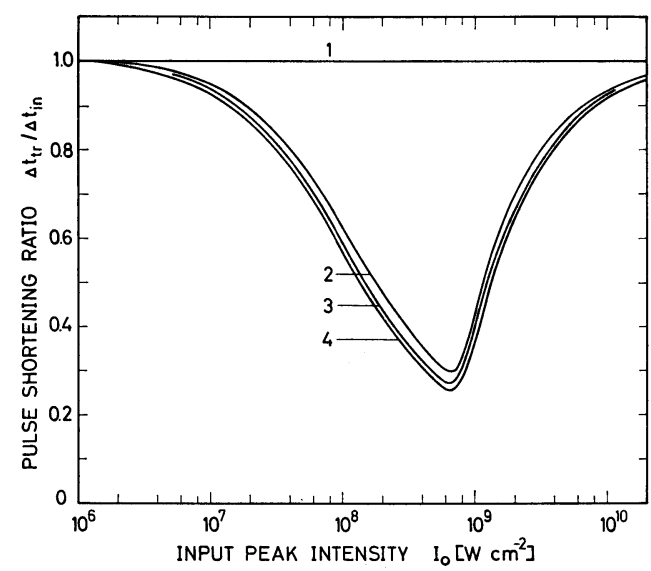

Figure 6 Pulse shortening ratio $\Delta t_{\mathrm{tr}} / \Delta t_{\mathrm{in}}$ as a function of input peak intensity $I_{0}$ for several incident pulse shapes in the stationary case. 1: rectangular, 2: Gaussian, 3: hyperbolic secant, 4: Lorentzian pulse shape. $\left(T_{0}=10^{-7}, I_{\mathrm{s}}=5.6 \times 10^{7} \mathrm{~W} \mathrm{~cm}^{-2}\right.$. $)$

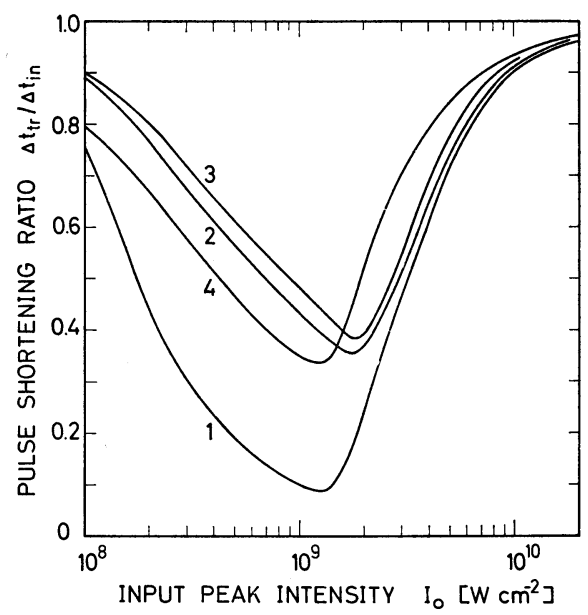

Figure 7 Pulse shortening ratio $\Delta t_{\mathrm{tr}} / \Delta t_{\mathrm{in}}$ as a function of input peak intensity $I_{0}$ for several incident pulse shapes in the transient situation $\Delta t_{\text {in }} / \tau=0.88\left(\Delta t_{\text {in }}=8 \mathrm{ps}, \tau=9.1 \mathrm{ps}\right) .1$ : rectangular, 2: Gaussian, 3: hyperbolic secant, 4: Lorentzian pulse shape. $\left(T_{0}=10^{-7}\right.$.)

(Gaussian input pulses). The dash-dot lines indicate the peak intensity reduction at $I_{0, \text { opt }}$ (most effective pulse shortening). Fig. 8 demonstrates quite clearly, that the peak intensity of the input pulse is only moderately reduced for $I_{0} \gtrsim I_{0}$,opt, but severe absorption occurs for $I_{0}<I_{0}$,opt. For instance, at $T_{0}=10^{-7}$ and $I_{0}=I_{0, o p t}$ the peak intensity of the transmitted pulse $I_{\mathrm{tr} \text {, max }}$ is approximately $30 \%$ of the input peak intensity $I_{0}$.

The total energy of the pulse is reduced by a larger factor than the peak intensity: The beam cross-section is narrowed, the temporal profile is shortened and the peak intensity is reduced. In Figs. $9 \mathrm{a}$ and $\mathrm{b}$ the energy transmission $T_{\mathrm{E}}=E_{\mathrm{tr}} / E_{\mathrm{in}}$ is plotted 


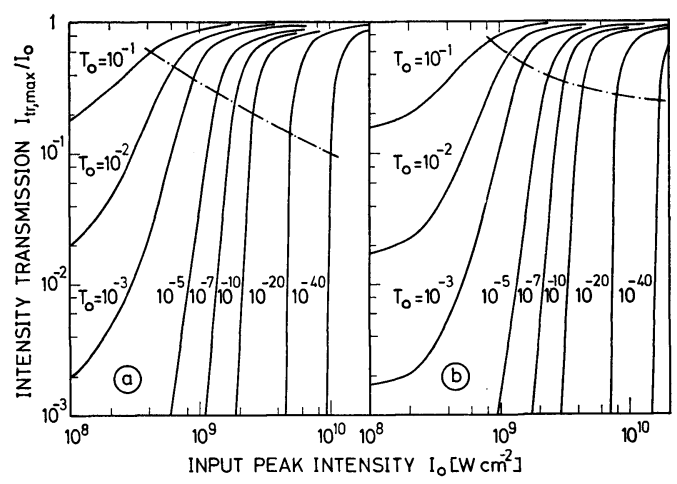

Figure 8 Normalized peak intensity $I_{\mathrm{tr}, \max } / I_{0}$ after a single pass through a saturable absorber as a function of input peak intensity $I_{0}$ for several values of $T_{0}$. Gaussian shape of the input pulse, $\Delta t_{\text {in }}=$ $8 \mathrm{ps}$ (a) and $\Delta t_{\mathrm{in}}=4 \mathrm{ps}(\mathrm{b})$. The dash-dot line indicates $I_{\mathrm{tr} \text {, max }}$ for $I_{\mathrm{o}, \mathrm{opt}}$ (most effective pulse shortening).

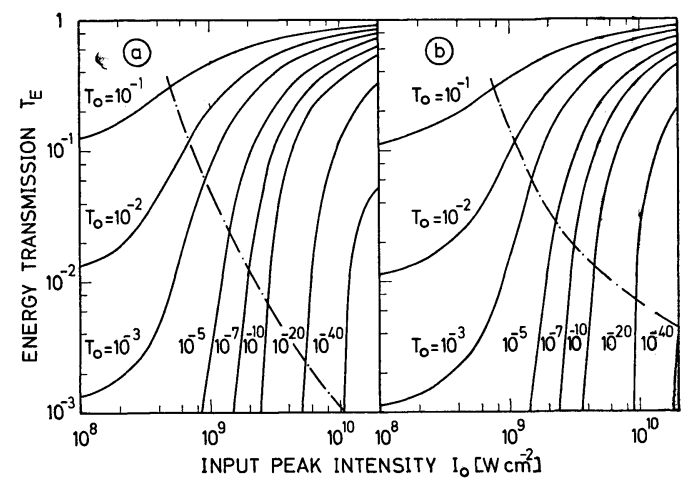

Figure 9 Energy transmission $T_{\mathrm{E}}$ of a spatial and temporal Gaussian input pulse through a saturable absorber as a function of input peak intensity $I_{0}$ for several values of $T_{0}$. Input pulse duration $\Delta t_{\text {in }}=8 \mathrm{ps}$ (a) and $\Delta t_{\text {in }}=4 \mathrm{ps}(\mathrm{b})$. The dash-dot line indicates $T_{\mathrm{E}}$ at $I_{0, \text { opt. }}$.

as a function of the input peak intensity for various values of $T_{0}$. (Pulses with spatial and temporal Gaussian shape of 8 and 4 ps duration.) Concerning the calculations of Fig. 9 see [20]. It is readily seen from Figs. $9 \mathrm{a}$ and $\mathrm{b}$ that the energy transmission at $I_{\mathrm{o}, \mathrm{opt}}$ (dash-dot line) changes from $T_{\mathrm{E}} \simeq 0.3$ at $T_{\mathrm{o}}=0.1$ to $T_{\mathrm{E}} \simeq 10^{-3}$ at $T_{\mathrm{o}}=10^{-40}$.

In our experiments we were able to shorten pulses from 8 to $2.6 \mathrm{ps}$ with a single dye cell (see Fig. 4a). More drastic pulse shortening to the subpicosecond range was achieved by multiple passes through absorbers and subsequent amplifiers as described in the next subsection.

\subsection{Multiple passes through an absorber-amplifier system}

The experimental set-up for pulse shortening by multiple passes through an absorberamplifier system is depicted schematically in Fig. 10. The selected pulse from a modelocked Nd-glass laser traverses four times the absorber-amplifier system consisting of two dye cells DC1 and DC2 and a Nd-glass amplifier. In an additional dye cell DC3 


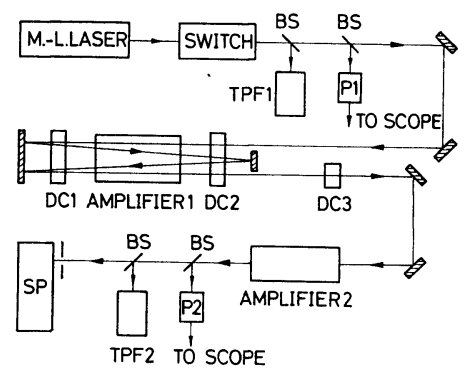

Figure 10 Experimental system for multiple transits through the absorber-amplifier system. Beam splitters BS; photodetectors P1, P2; two-photon fluorescence systems TPF1, TPF2; absorber cells DC1 $\left(T_{0}=1.4 \times 10^{-2}\right), \mathrm{DC} 2\left(T_{0}=1.4 \times 10^{-2}\right), \mathrm{DC} 3\left(T_{0}=7 \times 10^{-3}\right) ;$ spectrograph SP.

and a second amplifier, the pulse is shortened once more and increased in energy. The pulse duration of the incident and the final pulse is monitored by the two-photon fluorescence systems TPF1 and TPF2, respectively. The spectrum of the transmitted pulse is measured with a grating spectrograph. We have performed a series of experiments. The most relevant data are as follows. With $T_{\mathrm{o}}=1.4 \times 10^{-2}$ in DC1 and DC2, $T_{\mathrm{o}}=7 \times 10^{-3}$ in DC3 and with an amplification factor $\gamma$ of approximately $4.1 \mathrm{per}$ pass through amplifier 1 we observed a pulse shortening from 8 to $\simeq 0.7 \mathrm{ps}$ and from 5 to $\simeq 0.5 \mathrm{ps}$. The measured spectral distribution of the transmitted pulse had a half width of $\Delta \tilde{\nu}_{\text {out }} \simeq 30 \mathrm{~cm}^{-1}$ (FWHM). This observation indicates that our shortened pulses are bandwidth limited $\left(\Delta \nu_{\text {out }} \times \Delta t_{\text {tr }} \simeq 0.6\right)$ [10]. It is interesting to emphasize that we worked with a total absorber transmission of $T_{0, \text { tot }} \simeq 10^{-17}$ and a total amplification factor of $\gamma_{\text {tot }} \simeq 300$. Under these conditions the pulses were shortened by a factor of ten to twelve. In a single pass the same total transmission would give a shortening factor of four at optimum input intensity. The absorber-amplifier system is more effective than a single absorber cell since the pulse is repeatedly amplified to the optimum intensity $I_{0, \text { opt. }}$.

The pulse development in the absorber-amplifier system was calculated for three typical examples as shown in Figs. 11a, b and c. The normalized intensity is plotted as a function of normalized time. In these calculations a Gaussian input pulse of $\Delta t_{\text {in }}=8 \mathrm{ps}$ and an initial peak intensity of $I_{0}=1.1 \times 10^{9} \mathrm{~W} \mathrm{~cm}^{-2}$ was assumed [22]; a transmission of $T_{0}=2 \times 10^{-4}$ per pass and amplification factors of $\gamma=4.0,4.1$ and 4.2 were used in the Figs. 11a, b, and c, respectively. The broken line represents the Gaussian input pulse, curve 1 indicates the pulse after the first passage through the absorber, curve 2 shows the same pulse after it was amplified and has passed through a second absorber. Curve 4 gives the final pulse which has traversed five absorber cells with four amplifications in between. At low amplification values ( $\gamma \leq 4$ in our example) the pulse becomes strongly absorbed in each absorber cell and decreases strongly (see Fig. 11a). At high amplification $(\gamma \geq 4.2)$ the outgoing pulse exceeds the intensity of the incoming pulse (see Fig. 11c; the amplification is larger than the reduction of peak intensity in the dye). At an amplification factor of $\gamma \simeq 4.1$ the pulse is most effectively shortened. The calculations of Fig. 11 demonstrate the strong effect of the 


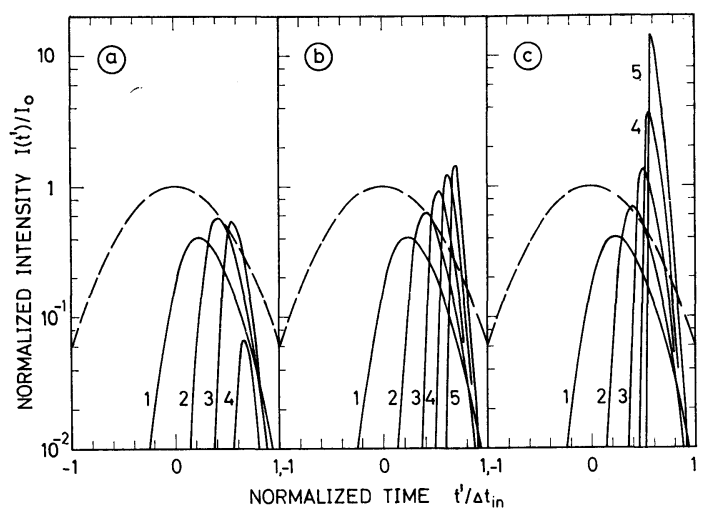

Figure 11 Calculated pulse development for five transits through an absorber-amplifier system. Broken curve: Gaussian input pulse of $\Delta t_{\mathrm{in}}=8 \mathrm{ps}$. Solid curves: intensity distribution of the pulse after 1 to 5 passes through absorbers (and 0 to 4 transits through amplifiers). Absorber transmission per mass: $T_{0}=2 \times 10^{-4}$. Amplifier gain per pass: $\gamma=4.0(\mathrm{a}), \gamma=4.1(\mathrm{~b})$, and $\gamma=4.2$ (c).

amplification factor quite vividly. In practice it is important to adjust the gain carefully to obtain the desired performance of the absorber-amplifier system.

It should be emphasized that various non-linear processes occur when the peak intensity of the pulse exceeds $5 \times 10^{9} \mathrm{~W} \mathrm{~cm}^{-2}$. At these intensities, for instance, twophoton absorption in the Nd-glass rod has been observed [23]. In this case the centre of the pulse experiences more non-linear absorption than the wings of lower intensity; as a result the pulse broadens in the Nd-glass amplifier. With a beam expanding telescope the pulse intensity in the amplifier is readily held below $5 \times 10^{9} \mathrm{~W} \mathrm{~cm}^{-2}$.

\section{Limitations of the pulse shortening technique}

In concluding this paper we wish to make several remarks concerning the shortest pulses, which might be generated in an absorber-amplifier system.

(i) At higher peak intensities and for many transits non-linear processes such as self-focusing [24], self-phase modulation [8] and dispersion [9] effects have to be considered. These effects increase with the length of the non-linear media (Nd-glass and dye) and limit the total length of the optical path.

(ii) The amplifier broadens the pulse when the inverse of the pulse duration approaches the bandwidth of the active medium (the central frequencies are preferentially amplified). For Nd-glass the spectral bandwidth is $\Delta \tilde{\nu}_{\mathrm{L}} \simeq 200 \mathrm{~cm}^{-1}$ [3], i.e. pulses with $\Delta t \lesssim 0.5 /$ $\Delta \nu_{\mathrm{L}} \simeq 10^{-13} \mathrm{~s}$ should be broadened.

(iii) When the pulse duration becomes shorter than the dephasing time $T_{2}$ of the amplifier or of the dye, coherent processes (self-induced transparency) have to be considered [25, 2]. For Nd-glass the dephasing time is $T_{2}$ (amplifier) $=1 /\left(\pi \Delta \nu_{\mathrm{L}, \mathrm{hom}}\right) \simeq$ $0.6 \mathrm{ps}$ at room temperature [3]. The area under the electric field

$$
\theta=(\mu / \hbar) \int_{-\infty}^{\infty} E \mathrm{~d} t
$$

is a characteristic parameter for pulses shorter than $T_{2}$. In our case, $\theta$ is less than 0.3 
for the amplifier $\left(I<10^{10} \mathrm{~W} \mathrm{~cm}^{-2}, \Delta t \lesssim 0.6 \mathrm{ps}\right.$, electric dipole moment $\mu \simeq 2 \times 10^{-29}$ $\mathrm{Ccm}$ [26]). Under these conditions the amplifier gain is expected to be constant without breakup of the light pulse when the pulse duration becomes shorter than $T_{2}$ (amplifier) $[2,25]$.

(iv) The transverse relaxation time of the saturable absorber is $T_{2}$ (dye) $\simeq 10^{-13} \mathrm{~s}$ [17] and the dipole matrix element is $\mu$ (dye) $\simeq 2.5 \times 10^{-27} \mathrm{Ccm}$ [28]. When self-induced transparency occurs in the absorber (at $\Delta t<T_{2}$ (dye) $\simeq 10^{-13} \mathrm{~s}$ ) the medium does not act as a saturable dye $[29,30]$; the rate equations used in this paper are not any longer applicable.

In summary the shortest pulse duration obtainable with the method described above is expected to be approximately $10^{-13} \mathrm{~s}$.

\section{Acknowledgement}

The author gratefully acknowledges valuable collaboration with Professor Dr W. Kaiser, Dr A. Laubereau and Dr D. von der Linde.

\section{References}

1. A. J. DemARIA, W. H. GLENN, JUn., M. J. BRIENZA and M. E. MACK, Proc. IEEE 57 (1969) 2-25.

2. P. G. KRYUKOV and V. S. LETOKHOV, Sov. Phys. Uspekhi 12 (1970) 641-672.

3. e. SNitZer, Appl. Opt. 5 (1966) 1487-1499. e. SNitZer and C. G. Young, 'Lasers', Vol. 2, Ch. 2 (Ed. A. K. Levine), Marcel Dekker, New York, 1968.

4. W. R. sooy, Appl. Phys. Lett. 7 (1965) 36-37. Attempts have been made to reduce the spectral narrowing with a Fabry-Perot interferometer, see M. W. GEOCH, Opt. Comm. 7 (1963) 116-120; and D. VON LINDE and K. F. RODGERS, Opt. Comm. 8 (1973) 91-94.

5. B. YA. ZEL'DOVICH and T. I. KUZNETSOVA, Sov. Phys. Uspekhi 15 (1972) 25-44.

6. P. G. KRYUKOV and v. S. LETOKHOV, IEEE J. Quant. Electr. QE-8 (1972) 766-782.

7. E. B. TREACY, Appl. Phys. Lett. 17 (1970) 14-16.

8. M. A. DUGUAY, J. W. HANSEN and S. L. SHAPIRO, IEEE J. Quant. Electr. QE-6 (1970) 725-743.

9. R. R. CUBEDdu and o. SVelto, IEEE J. Quant. Electr. QE-5 (1969) 495-502. o. Svel to, Opt. Comm. 3 (1971) 105-106.

10. D. VON DER LINDE, IEEE J. Quant. Electr. QE-8 (1972) 328-338.

11. E. B. TREACY, Physics Letters 28A (1968) 34-35.

12. A. LAUbereau, ibid 29A (1969) 539-540. A. LAUbereau and D. von Der linde, $Z$. Naturf. 25a (1970) 1626-1642.

13. M. J. COlles, Appl. Phys. Lett. 19 (1971) 23-25.

14. A. PENZKOFER, D. VON DER LINDE, A. LAUBEREAU and W. KAISER, ibid 20 (1972) 351-354. For later related work see P. G. KRYUKOV, YU. A. MATVEETS, S. V. CHEKALIN and O. B. ShatVerashili, Sov. Phys. Jetp. Letters 16 (1972) 81-83.

15. The experimental results of reference [20] confirm that the dye used can be accounted for by a two-level system.

16. In our experiments the characteristic parameters of the dye are: $\tau=9.1 \mathrm{ps}$ and $\sigma=1.84 \times 10^{-16}$ $\mathrm{cm}^{2}$; see Eastman Kodak dye No. A9860 data release.

17. M. Her Cher, Appl. Opt. 6 (1967) 947-954.

18. Steady state shortening of nanosecond light pulses $\left(\Delta \mathrm{t}_{\text {in }} \gg \tau\right)$ with an absorber-amplifier system has been reported by N. G. BASOV, P. D. KRYUKOV, V. S. LETOKHOV and YU. A. MATVEETS, Sov. Phys. Jetp. 29 (1969) 830-835.

19. J. D. MACOMber, J. Appl. Phys. 38 (1967) 3525-3530.

20. A. Penzkofer, D. von Der linde and A. laubereau, Opt. Comm. 4 (1972) 377-379. 
21. J. A. giordmaine, P. M. Rentzepis, s. L. Shapiro and k. w. Wecht, Appl. Phys. Lett. 11 (1967) 216-218.

22. The shape of the initial pulses is nearly Gaussian in our experiments; see D. VON DER LINDE and a. laubereau, Opt. Comm. 3 (1971) 279-283.

23. A. PEnZKOFER and W. KAISER, Appl. Phys. Letters 21 (1972) 427-430.

24. S. A. AKHMANOV, R. V. KHOKHLOV and A. P. SUKHORUKOV, 'Laser Handbook', Vol. 2 Ch. E3 (Ed. F. T. Arecchi and E. O. Schulz-DuBois) North Holland Publ. Co., Amstere dam, 1972

25. s. L. McCall and E. L. HAHN, Phys. Rev. 183 (1969) 457-485.

26. $\mu$ (amplifier) is calculated from the spontaneous relaxation time $\tau_{\mathrm{sp}}$ of the upper laser level $\left(\tau_{\mathrm{sp}} \simeq 6.4 \times 10^{-4} \mathrm{~s}\right.$ for Schott laser glass LG630 used in our experiments). The relation $1 / \tau_{\mathrm{sp}}=16 \pi^{3} \nu_{\mathrm{L}}^{3} \eta|\mu|^{2} /\left(3 h c^{3} \epsilon_{0}\right)$ has been used; see e.g. reference [27], page 183 .

27. R. H. PANTELl and H. E. PUthofF, 'Fundamentals of Quantum Electronics', John Wiley \& Sons Inc., New York, 1969.

28. $\mu$ (dye) is estimated from the absorption cross-section $\sigma$ of the saturable absorber [16], using the relation $\sigma=4 \pi^{2} \nu\left[\left(\eta^{2}+2\right) / 3\right]^{2}|\mu|^{2} \mathrm{~T}_{2} /(3 h c \eta)$; see e.g. reference [27], page 60 .

29. A. C. Selden, J. Phys. D: Appl. Phys. 3 (1970) 1935-1943.

30. K. W. SMith and L. Allen, Opt. Comm. 8 (1973) 166-170. 